\title{
Can CPAP prevent the need for endotracheal intubation in patients with hypoxemia after abdominal surgery?
}

Squadrone V, Coha M, Cerutti E, Schellino MM, Biolino P, Occella $\mathrm{P}$, et al. Continuous positive airway pressure for treatment of postoperative hypoxemia. A randomized controlled trial. JAMA 2005; 293:589-95.

Background: Hypoxemia is estimated to complicate $30 \%-50 \%$ of postoperative abdominal surgery cases, and respiratory failure requiring endotracheal intubation may occur in $8 \%-$ $12 \% .{ }^{1,2}$ Continuous positive airway pressure (CPAP) has been shown to improve oxygenation after abdominal surgery (presumably by reducing postoperative atelectasis), ${ }^{3,4}$ but no trials have shown a reduction in more meaningful clinical outcomes in these patients.

Design: In this prospective, multicentred, randomized controlled trial, patients with hypoxemia (ratio of arterial oxygen tension to inspiratory oxygen fraction $\left[\mathrm{PaO}_{2} / \mathrm{FiO}_{2}\right] \leq 300$ ) following elective laparotomy with general anesthesia were randomly assigned to receive oxygen for 6 hours (at $\mathrm{FiO}_{2}$ of 0.5 ) either by Venturi mask (control group) or by CPAP of $7.5 \mathrm{~cm} \mathrm{H}_{2} \mathrm{O}$. Every 6 hours, patients in both groups were screened for resolution of their hypoxemia $\left(\mathrm{PaO}_{2} / \mathrm{FiO}_{2}\right.$ ratio $>300)$. The primary end point was need for endotracheal intubation, which was performed according to prespecified criteria, including severe hypoxemia (arterial oxygen saturation $<80 \%$ despite maximal $\mathrm{FiO}_{2}$ ). The ultimate decision to intubate was made by the attending clinician. Secondary end points included intensive care unit (ICU) and hospital lengths of stay, incidence of pneumonia, infection and sepsis, $\approx$ and hospital mortality. Anesthe$\approx$ sia, postoperative pain control, ventilation parameters and respiratory physiotherapy were controlled with protocols.

Results: The trial was stopped after the first interim analysis
( $n=209)$ because the intubation rate in the oxygen plus CPAP group was lower than in the oxygen only group (1\% [1/105] v. $10 \%$ [10/104], $p=0.005)$. Severe hypoxemia was the indication for intubation in 9 of the 11 patients. Several secondary end points also favoured the CPAP group, including reduced incidence of pneumonia, infection and sepsis. There were no significant differences between the groups in hospital mortality or in ICU or hospital lengths of stay.

Commentary: This well-designed trial showed that CPAP reduces the need for intubation and mechanical ventilation in patients with hypoxemia after abdominal surgery. Strengths of the study included strategies to minimize the inherent potential for bias given its unblinded design (e.g., use of prespecified intubation criteria and standardized protocols to control for potential confounders). The study's generalizability is limited by its restrictive inclusion criteria (e.g., no cardiovascular, pulmonary, malignant, infectious or severe renal disease, no obesity, and no facial, neck or chest wall abnormalities). Furthermore, most CPAP delivery systems in Canada involve a face or nasal mask. This trial used a may have contributed to the unusually low rate (4\%) of intolerance to therapy.

Administration of CPAP requires close supervision in a monitored setting, and routine delivery of CPAP in postoperative units would necessitate significant changes in training and staffing. It should also be noted that the primary end point (intubation) occurred relatively infrehelmet for CPAP delivery, which quently $(n=11)$, and just a few more events in the CPAP group would have rendered the results statistically nonsignificant. Prevention of endotracheal intubation is clearly desirable, but associated reductions in more convincing end points (e.g., ICU length of stay and hospital mortality) were not observed.

Practice implications: This study demonstrates that the use of CPAP to treat postoperative hypoxemia probably decreases the need for intubation and mechanical ventilation and appears to be safe. The potential benefits of this treatment will need to be weighed against practical implications for training, staffing and resources. The helmet system used to provide CPAP in this study is currently not widely available in Canada, and delivery of CPAP using face or nasal masks may not be as well tolerated by patients.

\section{Ryan Foster}

Damon C. Scales

Department of Critical Care

St. Michael's Hospital

Interdepartmental Division

of Critical Care

University of Toronto

Toronto, Ont.

\section{References}

1. Arozullah AM, Daley J, Henderson WG, Khuri SF. Multifactorial risk index for predicting postoperative respiratory failure in men after major noncardiac surgery. The National Veterans Administration Surgical Quality Improvement Program. Ann Surg 2000;232:242-53.

2. Thompson JS, Baxter BT, Allison JG, Johnson FE, Lee KK, Park WY. Temporal patterns of postoperative complications. Arch Surg 2003;138: 596-602.

3. Stock MC, Downs JB, Cooper RB, Lebenson IM, Cleveland J, Weaver DE, et al. Comparison of continuous positive airway pressure, incentive spirometry, and conservative therapy after cardiac operations. Crit Care Med 1984;12:969-72.

4. Lindner KH, Lotz P, Ahnefeld FW Continuous positive airway pressure effect on functional residual capacity, vital capacity and its subdivisions. Chest 1987;92:66-70. 